hep-th/9803087

UCSD/PTH 98-09

\title{
Supersymmetric gauge theories with a free algebra of invariants
}

\author{
Gustavo Dotti, Aneesh V. Manohar, and Witold Skiba \\ Department of Physics, University of California at San Diego, \\ 9500 Gilman Drive, La Jolla, CA 92093
}

\begin{abstract}
We study the low-energy dynamics of all $\mathcal{N}=1$ supersymmetric gauge theories whose basic gauge invariant fields are unconstrained. This set includes all theories whose matter Dynkin index is less than the index of the adjoint representation. We study the dynamically generated superpotential in these theories, and show that there is a $W=0$ branch if and only if anomaly matching is satisfied at the origin. An interesting example studied in detail is $S O(13)$ with a spinor, a theory with a dynamically generated $W$ and no anomaly matching at the origin. It flows via the Higgs mechanism to $S U(6)$ with a three-index antisymmetric tensor, a theory with a $W=0$ branch and anomaly matching at the origin.
\end{abstract}

\footnotetext{
${ }^{1}$ Address after April 1, 1998: FaMAF, Universidad Nacional de Córdoba, (5000) Córdoba, Argentina. e-mail: gdotti@fis.uncor.edu
} 


\section{Introduction}

The first step in studying the low-energy dynamics of supersymmetric gauge theories [1 3] is determining the structure of the moduli space. The moduli space can always be described in terms of the expectation value of composite fields which are gauge invariant polynomials constructed out of the microscopic chiral matter superfields [4] [6]. In general, the gauge invariant polynomials are subject to constraints. One can always choose a basic set of linearly independent composite fields such that all gauge invariant composites can be written as polynomials in the basic set. All constraints among gauge invariant composites are then reduced to nonlinear constraints among the basic set of invariants. Given the description of the classical moduli space, one can use symmetry arguments, the semi-classical limit, and various deformations of the theory to learn about the quantum behavior. Deformations such as adding mass terms or breaking the gauge group by the Higgs mechanism are particularly useful, and one can often use them to relate one theory to another theory whose low-energy description is already known.

In this article we examine all asymptotically-free theories with a free algebra of invariants. We consider theories with simple gauge groups and no tree-level superpotentials. By a free algebra of invariants we mean that the full classical moduli space is described in terms of independent gauge invariants which are not subject to any constraints. In a previous paper [7] a class of free-algebra theories was studied in which flavor anomalies are saturated by the basic gauge invariants. It was shown that, in most cases, matching of anomalies together with the requirement of a free algebra implied the existence of a branch of the theory with no dynamically generated superpotential. In this paper, we extend the results of Ref. [0] to all theories with a free algebra of invariants.

We expect different explanations for the lack of anomaly matching between the microscopic theory and the composites parameterizing the moduli space depending on the relative value of the Dynkin index of the matter fields, $\mu$, and the Dynkin index of the adjoint, $\mu_{a d j}$. When $\mu<\mu_{a d j}$, a dynamically generated superpotential is allowed by symmetries. Such a superpotential lifts the classical moduli space leaving no stable vacuum state. We explicitly check that in all cases where anomalies do not match a superpotential is indeed dynamically generated. Maximally breaking the original gauge group often leaves a pure Yang-Mills theory with gauge group $G_{P}$, and gaugino condensation in this minimal unbroken subgroup generates a dynamical superpotential. When the gauge group is completely broken, one can check that instanton contributions are responsible for generating a dynamical superpotential. There is an interesting difference between those theories with $\mu<\mu_{\text {adj }}$ where anomalies do match at the origin, and those where they do not. In theories where the anomalies match at the origin,the minimal unbroken gauge group $G_{P}$ is a product group, with identical factors (e.g. $S U(3) \times S U(3))$. The dynamical superpotential is generated by gaugino condensation in the components of $G_{P}$, leading to a multi-branched theory. For at least one branch of the theory, the superpotential generated by the various factors cancels, leading to a branch 
with $W=0$ [7]. In theories where anomalies do not match at the origin, $G_{P}$ is such that it is impossible to have a $W=0$ branch, and the theory always has a non-zero dynamical superpotential. When $\mu=\mu_{a d j}$, all free algebra theories have unbroken $U(1)$ gauge symmetries in the bulk of the moduli space, a fact recently noticed in Ref. [8]. In this case, after including photons and sometimes also massless monopoles in the low-energy spectrum, anomalies are saturated at the origin. For $\mu>\mu_{a d j}$, we argue based on flows that the free algebra theories are in an interacting non-Abelian Coulomb phase at the origin of the moduli spaces. Theories with $\mu>,<$, or $=\mu_{\text {adj }}$ can only flow via the Higgs mechanism to other theories of the same type.

Free algebra theories have been classified in the mathematics literature [9, 10]. After selecting theories free of gauge anomalies it turns out that all $\mu<\mu_{\text {adj }}$ examples have no constraints among the basic invariants [7]. The requirement that the theory have no gauge anomalies is essential for the result, which is why this fact was not noticed earlier in the mathematics literature. We also recall little known theorems proven in the mathematics literature which are helpful in analyzing supersymmetric gauge theories. First, theories that have unbroken $U(1)$ 's in the bulk of the moduli space must have $\mu=\mu_{\text {adj }}$ [11]. Second, any theory with $\mu>\mu_{\text {adj }}$ has completely broken gauge group in the bulk of the moduli space [12].

In the next section, we analyze the low-energy dynamics of the free algebra theories. A particularly interesting example studied in detail is the $S O(13)$ gauge theory with a spinor. This is a theory with $W \neq 0$ which flows via the Higgs mechanism to a theory with a $W=0$ branch. A complete list of free algebra theories with $\mu<\mu_{\text {adj }}$ is presented in Appendix A. There, we also indicate patterns of gauge symmetry breaking for these theories. In Appendix B, we discuss more mathematical issues. We derive a formula for the dimension of the classical moduli space, which is found to be equal to the number of microscopic degrees of freedom minus the dimension of the gauge group plus the dimension of the smallest unbroken subgroup. In applying this formula, one needs to be careful about the possibility that the smallest unbroken subgroup at a D-flat point is not the same as the smallest unbroken subgroup preserved by an arbitrary field configuration. This possibility occurs for $S O(10)$ gauge group with a single spinor field and the $S U(2 N+1)$ theory with an antisymmetric tensor and $2 N-3$ antifundamentals.

\section{Free Algebra Theories}

The moduli space of supersymmetric gauge theories can be parameterized by the vacuum expectation values (VEVs) of gauge invariant polynomials made out of matter superfields. For any theory there is a minimal set of invariants. In general, the basic gauge invariants are not independent, but are subject to nonlinear constraints. The VEVs allowed by these constraints are in one-to-one correspondence with the D-flat configurations of the microscopic degrees of freedom. Some theories, however, have unconstrained basic gauge invariants. 
These theories are the subject of this paper. We give a precise definition of classical moduli space and free algebra of invariants in Appendix B, where we also explain how to calculate the dimension of the moduli space.

All theories with a free algebra of invariants together with their basic set of invariants are given in Refs. [9, 10]. We restrict our attention to physical theories, that is those which are free of gauge anomalies. Since the dynamics of the free algebra theories with anomaly matching between the microscopic fields and the basic gauge invariants was already studied [6], we now consider only cases where anomalies do not match. We present all the cases in the following sections, dividing them according to the kind of non-perturbative effects that take place.

We will frequently consider theories along a flat direction, which partially or completely breaks the gauge group. It is easy to see that the sign of $\mu-\mu_{a d j}$ is preserved under such Higgs flow. In fact, when $G$ is broken to a subgroup $H, n\left(\mu-\mu_{\text {adj }}\right)=\tilde{\mu}-\tilde{\mu}_{\text {adj }}$, where $n$ is an integer, $\mu-\mu_{a d j}$ is computed in $G$, and $\tilde{\mu}-\tilde{\mu}_{a d j}$ is computed in $H$. The value of $n$ depends on the embedding of $H$ in $G$, and it is the $G$ winding number of the unit $H$ instanton.

\section{$2.1 \mu<\mu_{a d j}$}

It is a straightforward, yet tedious, exercise to select all physical theories with $\mu<\mu_{\text {adj }}$ among the free-algebra models of Ref. [9, 10. We list all of these theories in Appendix A. For each theory we give a sample pattern of symmetry breaking and the smallest unbroken subgroup along a generic flat direction. It turns out that there are no other $\mu<\mu_{\text {adj }}$ theories besides the ones with a free algebra [7]. Such statement is not true for theories with gauge anomalies. For example, an unphysical $S U(N)$ theory with $(N+2)$ fields in the fundamental representation and no fields in the antifundamental representation has constraints among the basic gauge invariants. The basic gauge invariants are $B_{i_{1} \ldots i_{N}}=\operatorname{det} Q_{i_{1}} \ldots Q_{i_{N}}$, which obey the constraint equations $B_{i_{1} \ldots i_{N}} B_{i_{N+1} j_{1} \ldots j_{N-1}} \epsilon^{i_{1} \ldots i_{N+2}}=0$.

$U(1)$ global symmetries, including the R-symmetry, restrict the form of a dynamically generated superpotential. In terms of the microscopic matter fields, $\phi_{i}$, the superpotential can be written as [1]

$$
W_{d y n} \propto\left(\frac{\Lambda^{\left(3 \mu_{a d j}-\mu\right) / 2}}{\prod_{i} \phi_{i}^{\mu_{i}}}\right)^{\frac{2}{\mu_{a d j}-\mu}},
$$

where $\mu_{i}$ is the Dynkin index of the $i$-th representation and $\mu=\sum_{i} \mu_{i}$. Whenever $\mu<\mu_{\text {adj }}$ such a superpotential has a good classical behavior. $\Lambda$ occurs in the numerator, so that $W_{d y n} \rightarrow 0$ for large values of the fields. For $\mu=\mu_{a d j}, \mathrm{R}$ symmetry prohibits the generation of a superpotential. For $\mu>\mu_{a d j}$, a dynamical superpotential cannot be generated in the free algebra theories, since $\Lambda$ occurs in the denominator, and $W_{d y n}$ does not have the correct behavior for large values of the fields. In those cases where a quantum superpotential is allowed, one still needs to check whether or not it is actually generated by non-perturbative effects. One way of checking is by integrating out matter fields from theories with a larger 
number of flavors using the results of Refs. [8, 13 15]. This approach can frequently be very cumbersome as theories with additional matter fields tend to have a large number of basic gauge invariants and complicated superpotentials. Here, we take a different path. We explore points on the moduli space where the original gauge group is broken. The effective theory below the scale of the massive gauge bosons is frequently known, and is usually easier to analyze than the original theory.

All $\mu<\mu_{a d j}$ theories are listed in Appendix A. For those analyzed in Ref. [7] for which anomalies match at the origin, the smallest unbroken subgroup at generic points on the moduli space turns out to be a product group. The remaining pure Yang-Mills theory exhibits gaugino condensation and has superpotentials generated in each factor group. The branch with zero superpotential arises due to a cancellation between the contributions from different factor groups to the superpotential.

We now explain why anomalies do not match for the remaining theories. They are listed in Appendix A, and can be divided into three types: $(i)$ the gauge group is completely broken, (ii) it is broken to a simple group, (iii) it is broken to a semi-simple group. For example, $S U(N)$ with $N-1$ flavors is an example of case $(i)$; if the number of flavors is smaller than $N-1$, it is an example of $(i i)$. $S U(2 N+1)$ theory with an antisymmetric tensor and its conjugate is an example of (iii), since the gauge group is broken down to $S U(2)^{N}$ at generic points on the moduli space.

Inspecting the table in Appendix A we conclude that in case $(i)$ the Higgs flow always contains an $S U(2)$ group with two fields in the fundamental representation. The $S U(2)$ theory with two fundamentals has been shown by an explicit calculation to have a non-zero superpotential generated by instantons [1]. Using scale-matching relations we checked that this has to be the case for all other theories with completely broken gauge group at generic values of the moduli fields.

In case $(i i)$, when the maximal breaking leaves a simple group, we can also use the scale matching to show that there is a non-zero superpotential. From the study of supersymmetric QCD [3] and other simple groups with fundamental matter fields 16 18 we know that pure $\mathcal{N}=1$ Yang-Mills theories exhibit gaugino condensation, which is responsible for the generation of a superpotential. A large fraction of theories listed in Appendix A have a nonzero superpotential due to gaugino condensation in the unbroken subgroup. We comment on some examples from this class at the end of this section.

The remaining case is when semi-simple groups remain unbroken at the points of maximal breaking. Obviously, gaugino condensation takes place in each unbroken factor, but there is no guarantee that contributions to the superpotential from the different factor groups do not cancel. After all, this effect was responsible for vanishing superpotentials in theories with anomaly matching. There are only seven theories without anomaly matching which have unbroken product groups at generic points on the moduli space. These are $S O(14)$ with $2 \square+S, S O(14)$ with $\square+S, S O(13)$ with $\square+S, S O(13)$ with $S, S O(12)$ with $S+S^{\prime}, S U(6)$ 
with $\mathrm{\theta}+\square+\bar{\square}$ and $S U(2 N+1)$ with $\mathrm{\theta}+\overline{\mathrm{G}} \cdot \mathrm{l}$ (In the table in Appendix A, these theories are numbered 53,53,30,31, 52, 11 and 6 , respectively.) The behavior of the different theories is related, since there are flows between them along certain flat directions:

$$
\begin{aligned}
S O(14): 2 \square+S & \stackrel{\langle\square\rangle}{\longrightarrow} S O(13): \square+S \stackrel{\langle\square\rangle}{\longrightarrow} S O(12): S+S^{\prime} \stackrel{\langle S\rangle}{\longrightarrow} S U(6): \text { 日 }+\square+\bar{\square} \stackrel{\langle\square+\bar{\square}\rangle}{\longrightarrow} \\
S O(14): \square+S & \stackrel{\langle\square\rangle}{\longrightarrow} S O(13): S \stackrel{\langle S\rangle}{\longrightarrow} S U(3) \times S U(3),
\end{aligned}
$$

where we indicated the smallest unbroken subgroup as the last step of the flow. We only need to understand why non-zero superpotentials are generated in the $S U(2 N+1)$ theory with $\boxminus+\overline{\bar{B}}$ and the $S O(13)$ with a spinor; all other cases are explained by scale matching along flat directions.

It is interesting that $S U(2 N)$ with $\boxminus+\overline{\bar{B}}$ has a branch with a zero superpotential due to cancellations among gaugino condensates in the unbroken $S U(2)^{N}$ subgroup [7]. On the other hand, $S U(2 N+1)$ does not have a vanishing superpotential, even though it has the same $S U(2)^{N}$ unbroken subgroup. The VEV of the tensor fields which breaks $S U(2 N+1)$ to $S U(2)^{N}$ is

$$
\langle\boxminus\rangle=\langle\overline{\bar{G}}\rangle=\operatorname{diag}\left(v_{1} \sigma_{2}, v_{2} \sigma_{2}, \ldots, v_{N} \sigma_{2}, 0\right)
$$

The massive gauge bosons, which enter the scale matching, decompose under $S U(2)^{N}$ as the $2 \cdot(2,2,1, \ldots, 1)(+$ permutations $)$ and $2 \cdot(2,1,1, \ldots, 1)(+$ permutations $)$. The masses squared of the $(2,2, \ldots)$ bosons are proportional to $\left(v_{i}-v_{j}\right)^{2}$ for one set and to $\left(v_{i}+v_{j}\right)^{2}$ for the other. In case of the the $(2,1, \ldots)$ bosons the masses squared are proportional to $v_{i}^{2}$. Thus, the scale of the $i$-th $S U(2)$ factor is related to the scale $\Lambda$ of $S U(2 N+1)$ by

$$
\Lambda_{i}^{6} v_{i}^{2} \prod_{j \neq i}\left(v_{i}^{2}-v_{j}^{2}\right)^{2}=\Lambda^{4 N+4}
$$

and the dynamical superpotential is

$$
W=\sum_{i} \pm \Lambda_{i}^{3}
$$

For simplicity, let us examine the case of the $S U(7)$ group. The superpotential induced by gaugino condensation is

$$
W=\Lambda_{S U(7)}^{8}\left[ \pm \frac{1}{v_{1}\left(v_{1}^{2}-v_{2}^{2}\right)\left(v_{1}^{2}-v_{3}^{2}\right)} \pm \frac{1}{v_{2}\left(v_{2}^{2}-v_{1}^{2}\right)\left(v_{2}^{2}-v_{3}^{2}\right)} \pm \frac{1}{v_{3}\left(v_{3}^{2}-v_{1}^{2}\right)\left(v_{3}^{2}-v_{2}^{2}\right)}\right]
$$

\footnotetext{
${ }^{2} S$ and $S^{\prime}$ denote the spinor representations.
} 
which does not have a zero branch. For $S U(2 N) \rightarrow S U(2)^{N}$, there are no massive gauge bosons in the $(2,1, \ldots)$ representation, and matching involves only products of $\left(v_{i}^{2}-v_{j}^{2}\right)$. This combination leads to a cancellation, as pointed out in Ref. [7].

Now let us turn to the $S O(13)$ theory with one field $S$ in the spinor (64) representation. A particular VEV of the spinor field can break $S O(13)$ to an $S U(6)$ theory with one field in the three-index antisymmetric representation. This $S U(6)$ theory with $T=\boxminus$ has been studied in Refs. [7, 13] and it has two branches. One branch has $W=0$, another $W=$ $\Lambda_{S U(6)}^{5} / \sqrt{T^{4}}$. Since anomalies do not match in the $S O(13)$ theory, it must have a nonvanishing superpotential that somehow gives both $W=0$ and $W=\Lambda_{S U(6)}^{5} / \sqrt{T^{4}}$ branches along the flat direction which breaks $S O(13)$ to $S U(6)$.

Fortunately, patterns of symmetry breaking in $S O(13)$ group with a spinor field have been classified in Ref. 19. There are two gauge invariants in this theory: $X=S^{4}$ and $Y=S^{8}$, so there are two inequivalent flat directions. Let us parameterize these flat directions at the microscopic level by two inequivalent spinor expectation values $S_{1}$ and $S_{2}$. We describe the moduli space as a linear combination $\alpha S_{1}+\beta S_{2}$. In terms of these parameters $X=\alpha^{4}+\beta^{4}$ and $Y=\alpha^{4} \beta^{4}$ [19]. Depending on the relative values of $\alpha$ and $\beta$ there are different patterns of $S O(13)$ breaking. For generic values of $\alpha, \beta S O(13)$ breaks to $S U(3) \times S U(3)$ with no matter fields transforming under the unbroken gauge group. When $\alpha=0$ and $\beta \neq 0, S O(13)$ is broken to $S U(6)$ with . Finally, when $\alpha=\beta \neq 0, S O(13)$ is broken to $S U(3) \times G_{2}$ with one field transforming as $(1,7)$ under $S U(3) \times G_{2}$.

From the previous analysis [7] of $S U(6)$ with $\theta$ we know the superpotential in the $S U(3) \times S U(3)$ theory along a generic flat direction. The $S U(3) \times S U(3)$ is a product of two independent Yang-Mills theories in which gaugino condensation takes place. However, the superpotential has a relative minus sign between the two contributions from the $S U(3)$ factors [7]: $W=\omega^{i} \Lambda_{1}^{3}-\omega^{j} \Lambda_{2}^{3}$, where $\omega$ is the cube root of unity, $i, j=1,2,3$ and $\Lambda_{1,2}$ are the characteristic scales of each $S U(3)$ factor. Therefore, using scale matching in $S U(3) \times G_{2}$ with $(1,7)$ we get

$$
W=\omega^{i} \Lambda_{1}^{3}-\omega^{j}\left(\frac{\Lambda_{2}^{11}}{Q^{2}}\right)^{1 / 3}
$$

along the $\alpha=\beta \neq 0$ flat direction. In the above equation $Q$ denotes the 7 of $G_{2}$ and $\Lambda_{2}$ the scale of $G_{2}$. Knowing that $S O(13)$ is broken to $S U(3) \times G_{2}$ when $\alpha=\beta$, we can identify the field $Q$ in the $(1,7)$ representation of $S U(3) \times G_{2}$ with $\alpha-\beta$. We can also use the breaking of $S O(13)$ to $S U(6)$ and then to $S U(3) \times S U(3)$ to match $\Lambda_{1}$ with the $S O(13)$ scale: $\Lambda^{25}=\Lambda_{1}^{9} X Y^{3 / 2}$. Combining all of this information together we obtain the superpotential for the full $S O(13)$ theory

$$
W=\frac{\Lambda^{25 / 3}}{X^{1 / 3} Y^{1 / 2}}\left(\omega^{i}-\omega^{j} \frac{X^{2 / 3}}{\left(X^{2}-4 Y\right)^{1 / 3}}\right) .
$$


In the above superpotentials we explicitly display the cube roots of unity rather than hide them in the definition of the cube root. When $S O(13)$ is broken to $S U(6)$ at a scale much larger than that of $S U(6) \rightarrow S U(3) \times S U(3)$, then $\alpha \ll \beta$, so that $Y \ll X^{2}$. In this limit $W \approx\left(\Lambda^{25 / 3} / X^{1 / 3} Y^{1 / 2}\right)\left(\omega^{i}-\omega^{j}\right)$. Indeed this superpotential correctly reproduces the superpotential of the $S U(6)$ theory. When $i=j$ we obtain the zero branch, and for $i \neq j$ the branch where $W \propto \Lambda_{6}^{5} / \sqrt{T^{4}}$.

In the remainder of this section we consider again theories with a simple unbroken gauge group at generic points of the moduli space. Several cases require a more careful analysis due to the presence of instantons in the broken group. For a detailed discussion of this effect see Ref. [20]. [ W When a one-instanton configuration in the unbroken subgroup does not correspond to one instanton in the original group there can be additional contributions to the superpotential from the instantons not represented in the unbroken subgroup. Among the

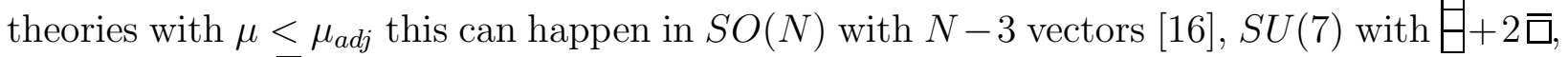
and $S p(6)$ with $\mathrm{B}+\square$. The particular flat directions for the $S U(7)$ and $S p(6)$ theories listed in Table 3 of Appendix A yield subgroups with the index of embedding equal to two. Both theories have an unbroken $S U(2)$ at generic points of their moduli spaces, thus one expects superpotentials generated by gaugino condensation. However, the instanton contribution from the broken part of the gauge group could potentially cancel the contribution arising due to gaugino condensation.

This is indeed what happens in the case of $S O(N)$ with $N-3$ vectors [16]. The $S O(N)$ theory has two branches. One branch has a dynamically generated superpotential, and another one has additional massless states at the origin of the moduli space. Those new massless particles are needed to saturate anomalies. We will argue that this does not happen for the $S U(7)$ and $S p(6)$ theories, which only have branches with non-zero dynamical superpotentials. One way of obtaining the superpotentials for these theories would be a direct computation of the coefficient of the instanton contribution to the superpotential. Instead, we explore different regions of the moduli spaces of these theories, where there are no non-trivial instanton configurations in the broken part of the group.

Apart from the breakings that we list in Table 3, there are other special points of enhanced symmetry on the moduli spaces of the $S U(7)$ and $S p(6)$ theories. The invariants for these theories were given in Ref. [14]. They are $B^{7}$ and $B^{3} \bar{Q}^{2}$ for the $S U(7)$ theory, $B^{4}$ and $B^{2} Q^{2}$ for $S p(6)$, where $B$ denotes the three-index antisymmetric tensor and $Q(\bar{Q})$ denote fields in the (a-)fundamental representation. The symmetry breaking with a non-trivial index of embedding arises when the fields $B$ obtain a VEV with the other fields having zero VEVs, which corresponds to nonzero values of $B^{7}, B^{4}$, respectively. The $S U(7)$ symmetry is also enhanced at points where $\left\langle B^{7}\right\rangle=0,\left\langle B^{3} \bar{Q}^{2}\right\rangle \neq 0$. Similarly $\left\langle B^{4}\right\rangle=0,\left\langle B^{2} \bar{Q}^{2}\right\rangle \neq 0$ is an enhanced symmetry point for $S p(6)$. Such choices of VEVs give the following unbroken

\footnotetext{
${ }^{3}$ We thank C. Csáki for discussions about this topic.
} 
subgroups

$$
\begin{aligned}
S U(7): \theta+2 \square & \rightarrow S p(6): \theta+\square, \\
S p(6): \theta+\square & \rightarrow S U(2) \times S U(2):(\square, \square) .
\end{aligned}
$$

These breakings do not have extra instanton contributions since the index of embedding is one. Moreover, since the $S U(7)$ theory flows to $S p(6)$, we need only to analyze the latter one.

We now argue that $S p(6)$ with $\Theta+\square$ has only branches with non-zero dynamicallygenerated superpotentials. Consequently, the same result applies to $S U(7)$. As we mentioned earlier, along the $B^{2} Q^{2}$ flat direction $S p(6)$ is broken to $S U(2) \times S U(2)$ with one field in the $(\square, \square)$ representation. Such a theory has been described in Ref. [21, and was found to have the superpotential $W=\left(\Lambda_{1}^{5 / 2} \pm \Lambda_{2}^{5 / 2}\right)^{2} / P^{2}$, where $P$ denotes the field in the $(\square, \square)$ representation, and $\Lambda_{i}$ 's are the scales of the $S U(2)$ groups. At the microscopic level, the VEV which breaks $S p(6)$ to $S U(2) \times S U(2)$ can be chosen to be $B_{245}=v, B_{346}=-v$ and $Q_{1}=v_{Q}$ with all other independent components of $B$ and $Q$ set to zero. $B_{245}$ and $B_{346}$ need to have opposite signs due to the tracelessness requirement for irreducible representations of $S p$. The unbroken $S U(2)$ 's act on the $(2,5)$ and $(4,6)$ indices of the original $S p(6)$. Of course, the D-flatness condition relates $v$ and $v_{Q}$, but we prefer to distinguish them for the time being.

Scales $\Lambda_{1,2}^{5}$ of the two $S U(2)$ factors have the same magnitude. They are, however, opposite in sign due to a discrete interchange symmetry of the two $S U(2)$ 's. A similar relative sign of two scales was found in an $S U(6)$ theory broken to $S U(3) \times S U(3) \mathbb{7}$. In terms of the VEVs and the $(\square, \square)$ field $B^{4}=v^{2} P^{2}, B^{2} Q^{2}=v^{2} v_{Q}^{2}$ and

$$
\frac{\Lambda_{S p}^{9}}{B^{4} \sqrt{B^{2} Q^{2}}}=\frac{\Lambda_{S p}^{9}}{P^{2} v^{3} v_{Q}}=\frac{\Lambda_{1,2}^{5}}{P^{2}}
$$

where the first term is the most general $S p(6)$ superpotential consistent with the symmetries. Under the interchange of the two $S U(2)$ 's we have $v_{Q} \rightarrow v_{Q}$, but $v \rightarrow-v$. Therefore, the two $\Lambda_{i}^{5}$ 's must differ by a minus sign. This means that the $S U(2)$ theories have a relative $\theta$ angle equal to $\pi$. Consequently, the superpotential generated in the unbroken $S U(2) \times S U(2)$ is $W=\Lambda_{S p}^{9}(1 \pm i)^{2} /\left(B^{4} \sqrt{B^{2} Q^{2}}\right)$, which does not vanish for either choice of the sign in the numerator. Thus, we have shown that the $S p(6)$ and $S U(7)$ theories have only branches with non-zero superpotentials. Since the form of the superpotential is unique in these theories, one can use the superpotential obtained this way and examine flat directions directions with potential instanton effects, thus obtain the coefficient of the instanton contribution in the broken part of the group. 


\section{$2.2 \mu=\mu_{a d j}$}

The list of all free algebra theories with $\mu=\mu_{\text {adj }}$ is quite short. It includes all theories with an adjoint superfield, which are automatically $\mathcal{N}=2$ Yang-Mills theories, and also $S O(N)$ with $N-2$ vectors, $S U(6)$ with 2 日 and $S p(6)$ with 2 日. One can check that anomalies are not saturated by the basic gauge invariants in any of these theories.

One can also check that at generic points of the moduli space there are only unbroken $U(1)$ gauge symmetries. All these theories are therefore in the Abelian Coulomb phase at low energies. Since the low-energy spectrum includes $U(1)$ photons their contribution has to be included in anomaly matching. Anomalies at the origin indeed match after including photons, the only exception being the $S O(N)$ theory with $N-2$ vectors, which has massless monopoles at the origin [16]. For all these theories the low-energy dynamics has been determined, namely the Seiberg-Witten curves have been found. For a list of references see [8].

It is interesting that the presence of unbroken $U(1)$ 's is specific to the $\mu=\mu_{\text {adj }}$ case. A theorem by Élashvili [11] states that for a simple group if there are unbroken $U(1)$ symmetries in the bulk of the moduli space then $\mu=\mu_{a d j}$. The bulk of the moduli space means here that the set of points where the gauge group is broken to a product of $U(1)$ 's is open (and dense) in the Zariski topology. We recall the definition of Zariski topology in Appendix B. The fact about $U(1)$ 's in the bulk of the moduli space was also noticed in Ref. \&].

As we already mentioned none of the free algebra theories with $\mu=\mu_{\text {adj }}$ has anomalies saturated by the basic gauge invariants. It is interesting that the same is true for the $\mu=\mu_{\text {adj }}$ theories with constraints. For the $\mu=\mu_{\text {adj }}$ constrained theories, the low-energy spectrum does consist of the basic gauge invariants, but some of the constraints are quantum modified. Due to the modification, some fields can be eliminated from the theory, and it may also happen that the modification excludes the origin from the quantum moduli space [3, 14, 15]. Note that for s-confining theories [13] integrating out fields such that the effective theory has $\mu=\mu_{a d j}$ always gives theories with a quantum modified moduli space.

\section{$2.3 \mu>\mu_{\text {adj }}$}

First, we want to point out a general result about $\mu>\mu_{\text {adj }}$ theories due to Andreev, Vinberg and Élashvili [12]. At a generic point of the moduli space the gauge group is completely broken, that is at most discrete symmetries remain unbroken. This theorem extends to all theories with semi-simple groups with $\mu>\mu_{a d j}$, not only the ones with a free algebra of invariants. For a gauge group which is a product of simple groups, the group is completely broken in the bulk of the moduli space if each factor satisfies $\mu>\mu_{\text {adj }}$.

The number of free-algebra theories with $\mu>\mu_{\text {adj }}$ is small. Those with anomaly matching were described in Ref. [7]. There are three remaining ones where anomalies do not match: $S U(N)$ with $\square+\bar{\square}, S p(6)$ with 2 日 and $S O(N)$ with $\mathrm{G}+\square$. We list the gauge invariants 


\begin{tabular}{|l|l|l|}
\hline$S U(N)$ & $\square+\square$ & $\operatorname{Tr}(S \bar{S})^{i}, i=1, \ldots, N-1$ \\
$S p(6)$ & 2 日 & $A_{1} A_{2} ; A_{1}^{3} A_{2}^{3} ; A_{1}^{i} A_{2}^{4-i}, i=0, \ldots, 4$ \\
$S O(2 N)$ & 日十ם & $A^{2 i}, i=1, \ldots, N-1 ; A^{2 k} Q^{2}, k=0, \ldots, N-1 ; A^{N}$ \\
$S O(2 N+1)$ & 日十ם & $A^{2 i}, i=1, \ldots, N ; A^{2 k} Q^{2}, k=0, \ldots, N-1 ; A^{N} Q$ \\
\hline
\end{tabular}

Table 1: Invariants of theories with $\mu>\mu_{\text {adj }}$ for which anomalies do not match at the origin. We indicate the gauge group in the first column, the field content in the second and the invariants in the last column. In writing the invariants, the fundamental representation is denoted by $Q$, symmetric tensors by $S$ and antisymmetric tensors by $A$. A subscript distinguishes between the two different $\mathrm{\theta}$.

of these theories in Table 1 for completeness.

As usual, we explore the moduli space of these theories. $S p(6)$ with 2 日 flows to $S U(3)$ with $\square+\bar{\square}$ after giving a VEV to the tensor fields. This is a particular case of $S U(N)$ with $\square+\bar{\square}$, which flows to an $S O(N)$ with $\square$ when one of the tensors gets a VEV. The $S O(N)$ theory with $\square$ has been recently discussed in Ref. [22]. The authors have argued that at the origin of the moduli space this theory is in a non-Abelian Coulomb phase. The same must then also be true for the $S U(N)$ with $\square+\square$ and $S p(6)$ with 2 日.

The remaining theory $S O(N)$ with $\mathrm{\theta}+\square$ is an example of a theory with a chiral superfield in the adjoint representation and additional matter fields, but no tree level-superpotential. The description of these kinds of theories is only known after the moduli space is restricted by adding a tree-level superpotential [23, 24].

\section{Conclusions}

We have studied all $\mathcal{N}=1$ theories without constraints among the basic gauge invariants. These include all theories with $\mu<\mu_{\text {adj }}$, theories in the Coulomb phase for $\mu=\mu_{\text {adj }}$ [8], and a few examples with $\mu>\mu_{\text {adj }}$. There are only two kinds of low-energy dynamics in all $\mu<\mu_{\text {adj }}$ theories. When anomalies match at the origin, the theory has a confining branch with no dynamical superpotential and branches with a dynamically-generated superpotential [7]. When anomalies do not match there is always a dynamically-generated superpotential, which excludes the origin from the quantum moduli space. We have checked that for each theory a superpotential is indeed generated. This task was accomplished by studying the flows of these theories along flat directions. We give a complete list of all $\mu<\mu_{\text {adj } j}$ theories together with a pattern of symmetry breaking that was helpful in analyzing each case in Appendix 


\begin{tabular}{|c|c|c|c|}
\hline & $\mu<\mu_{a d j}$ & $\mu=\mu_{a d j}$ & $\mu>\mu_{\text {adj }}$ \\
\hline Fi irred & $\overline{S O(14) S}$ & - & $\overline{S S O(N) \square}$ \\
\hline FA $\vee$ red & $S U(N)$ 日 $+\bar{\theta}$ & - & — \\
\hline FA $X$ \{irred & $S O(N) \square$ & $S U(N) a d j$ & 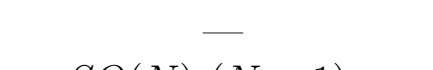 \\
\hline Th lred & $S U(N) \square+\bar{\square}$ & $S O(N)(N-2) \square$ & $S O(N)(N-1) \square$ \\
\hline $\mathrm{CN} \sqrt{ }\left\{\begin{array}{l}\text { irred } \\
\text { red }\end{array}\right.$ & - & - & $\operatorname{SU}(N)(N+1)(\square+\bar{n})$ \\
\hline y irred & - & - & $S O(15) S$ \\
\hline UN $X$ red & - & $S U(N) N(\square+\bar{\square})$ & $S U(N)(N+2)(\square+\bar{\square})$ \\
\hline
\end{tabular}

Table 2: Summary of all possible $\mathcal{N}=1$ theories with simple groups. The rows of the table divide theories according to the algebra of invariants ( $\mathrm{FA}=$ free algebra, $\mathrm{CN}=$ constraints), anomaly matching $(\sqrt{ })$ or lack of it $(X)$ and reducibility of the gauge representation of the microscopic field. We indicate by a dash that there are no examples of theories of a given kind. When there exist theories in a given class we give an example. $S$ indicates the spinor representation for $S O$ theories.

A. It is interesting that for most simple groups, the smallest unbroken subgroup is not restricted by the D-flatness condition. With the exception of $S O(10)$ with a spinor field and $S U(2 N+1)$ with $2 N-3$ antifundamentals, a generic field configuration preserves the same gauge symmetries as the most general D-flat configuration (see Appendix B).

Some results obtained by mathematicians aid in the understanding of the $\mu \geq \mu_{\text {adj }}$ cases. Theories with the $U(1)$ gauge bosons in the bulk of the moduli space can occur only when $\mu=\mu_{a d j}$. For $\mu>\mu_{\text {adj }}$ generic flat directions turn out to completely break the gauge group.

Table 2 summarizes all possibilities for $\mathcal{N}=1$ theories. The low energy dynamics of all theories with $\mu \leq \mu_{\text {adj }}$ is now understood. The last column in the table is mostly uncharted territory. There are only a few examples of theories whose dynamics is known to be described in terms of dual theories. Several examples with $\mu=\mu_{\text {adj }}+2$ are known to exhibit s-confinement. For all other $\mu>\mu_{\text {adj }}$ theories there is as yet no systematic analysis, and it is possible that new non-perturbative phenomena remain to be discovered.

\section{Acknowledgements}

We thank Ken Intriligator and Erich Poppitz for discussions. This work was supported in part by the U.S. Department of Energy under contract DE-FG03-97ER405046. 


\section{Appendix A Flows of $\mu<\mu_{a d j}$ theories}

All supersymmetric gauge theories with simple gauge group, zero tree-level superpotential and $\mu<\mu_{\text {adj }}$ have a free algebra of gauge invariant operators. In the table below we present all these theories, together with their Higgs flow along selected flat directions and the smallest unbroken subgroup. Theories are listed in the second column, and indicated by their gauge group followed by matter content and comments. For convenience, the theories are numbered in the first column, except for those theories with anomaly matching at the origin, for which the entry number has been replaced with $\mathbf{A}$, and those with a non-reductive stabilizer (Appendix B), by NR. The fourth column gives the unbroken subgroup and its field content along the flat direction associated with the field listed in the third column. We omit gauge singlets in the field content of the theories listed in the fourth column. TDF in the third column means that the only solution to the D-flatness condition is the trivial one. Since by breaking a $\mu<\mu_{\text {adj }}$ theory one arrives at another $\mu<\mu_{\text {adj }}$ theory, one can follow the pattern of symmetry breaking using the table. The entry number of the unbroken subgroup is listed in the fifth column. The final result of the the flow - the smallest unbroken subgroup - appears in the last column of the table.

Our notation is the following. $\phi_{k}$ denotes a $k$-index totally antisymmetric tensor (for $S p$ groups its highest weight irreducible component). For example, $\phi_{1}$ means the fundamental representation, $\phi_{2}$ means $\boxminus$, etc. $S, S^{\prime}$ denote the spinor representations, and an asterix indicates the conjugate representation. The variable $s_{i}$ takes integer values $1,2, \ldots, i$. When relevant, we indicate even numbers with subscript $e$, and the odd ones with $o$. If the smallest unbroken subgroup is trivial we indicate it by [ ]. It should also be understood that $S U\left(4-s_{3}\right)$ means [ ] when $s_{3}=3$, etc. The group isomorphisms $S O(6) \cong S U(4), S O(5) \cong$ $S p(4), S O(3) \cong S U(2)$ and the outer automorphisms of $S O(8)$ which permutes $\phi_{1}, S$ and $S^{\prime}$ were used to avoid redundant entries. In a few cases where the gauge group of a theory was omitted due to space limitations, it is understood to be the same as the group in the table entry right above it.

Table 3: All supersymmetric gauge theories with $\mu<\mu_{\text {adj }}$

\begin{tabular}{|l|l|c|l|c|l|}
\hline & $\mathbf{S U}(\mathbf{n})$ theories & $<>$ & Higgs flow & entry & SYM \\
\hline$(1)$ & $k\left(\phi_{1}+\phi_{1}^{*}\right): k<n$ & $\phi_{1}+\phi_{1}^{*}$ & $S U(n-1):(k-1)\left(\phi_{1}+\phi_{1}^{*}\right)$ & $(1)$ & $S U(n-k)$ \\
$(2)$ & $\phi_{2}+s_{2} \phi_{1}+\left(n-4+s_{2}\right) \phi_{1}^{*}$ & $\phi_{1}+\phi_{1}^{*}$ & $\phi_{2}+s_{2} \phi_{1}+\left(n-5+s_{2}\right) \phi_{1}^{*}$ & $(1,2)$ & $S U\left(3-s_{2}\right)$ \\
$(3)$ & $\phi_{2}+(n-4) \phi_{1}^{*}: n_{e}$ & $2 \phi_{1}^{*}+\phi_{2}$ & $S U(n-2): \phi_{2}+(n-6) \phi_{1}^{*}$ & $(3,32)$ & $S O(5)$ \\
$\mathbf{N R}$ & $\phi_{2}+(n-4) \phi_{1}^{*}: n_{o}$ & $2 \phi_{1}^{*}+\phi_{2}$ & $S U(n-2): \phi_{2}+(n-6) \phi_{1}^{*}$ & $(4,10)$ & $S U(5)$ \\
$(5)$ & $\phi_{2}+\phi_{2}^{*}+\phi_{1}+\phi_{1}^{*}$ & $\phi_{1}+\phi_{1}^{*}$ & $S U(n-1): \phi_{2}+\phi_{2}^{*}+\phi_{1}+\phi_{1}^{*}$ & $(1,5)$ & {[]} \\
\hline
\end{tabular}


Table 3: All supersymmetric gauge theories with $\mu<\mu_{\text {adj }}$

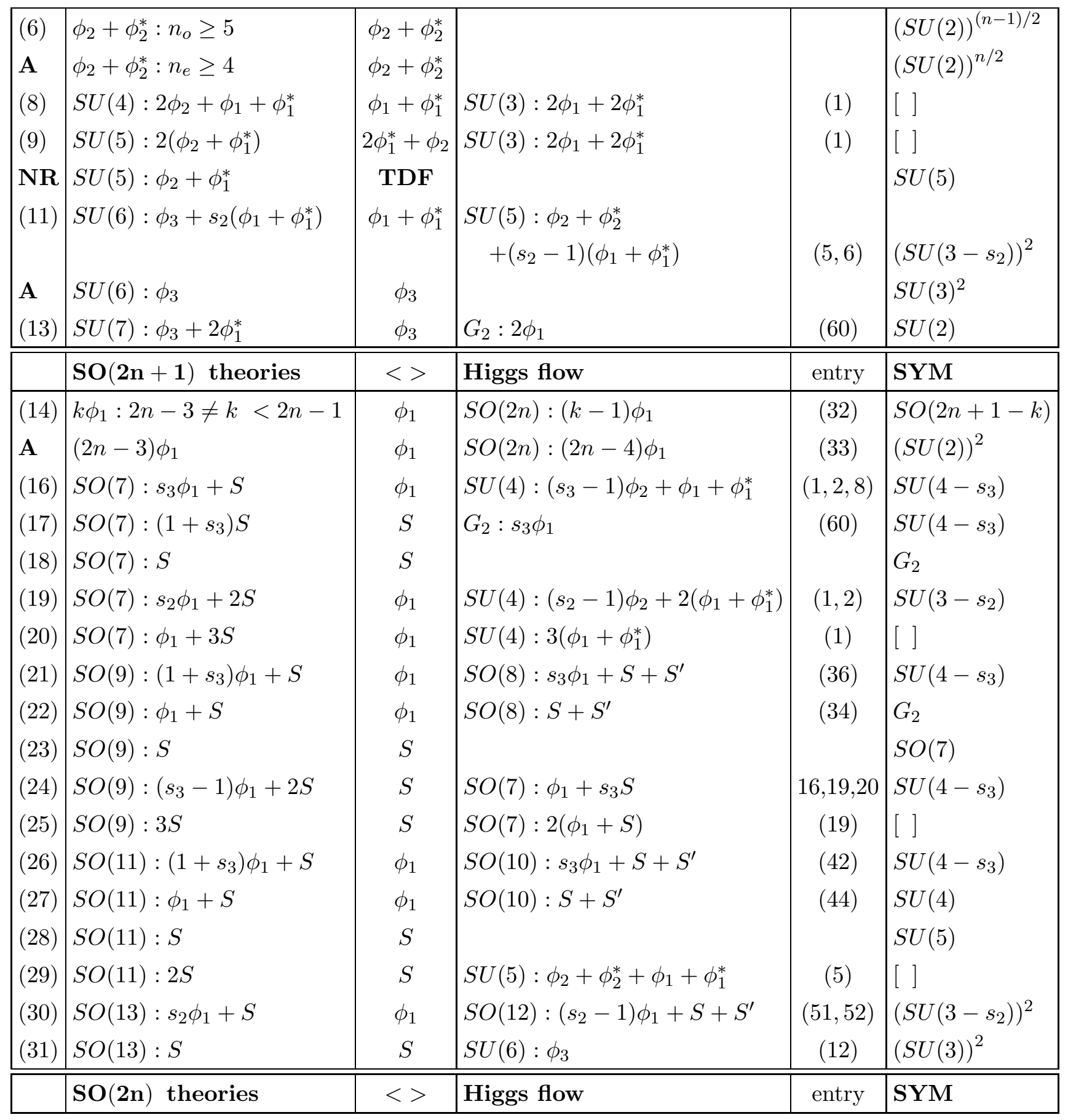


Table 3: All supersymmetric gauge theories with $\mu<\mu_{\text {adj }}$

\begin{tabular}{|c|c|c|c|c|c|}
\hline$(32)$ & $k \phi_{1}: 2 n-4 \neq k<2 n-2$ & $\phi_{1}$ & $S O(2 n-1):(k-1) \phi_{1}$ & (14) & $S O(2 n-k)$ \\
\hline $\mathbf{A}$ & $(2 n-4) \phi_{1}$ & $\phi_{1}$ & $S O(2 n-1):(2 n-5) \phi_{1}$ & $(15)$ & $(S U(2))^{2}$ \\
\hline$(34)$ & $S O(8): \phi_{1}+S$ & $\phi_{1}$ & $S O(7): S$ & (18) & $G_{2}$ \\
\hline$(35)$ & $\left(2-s_{2}+s_{3}\right) \phi_{1}+s_{2} S$ & $\phi_{1}$ & $S O(7):\left(1-s_{2}+s_{3}\right) \phi_{1}+s_{2} S$ & $(16,19)$ & $S U\left(4-s_{3}\right)$ \\
\hline$(36)$ & $S O(8): s_{3} \phi_{1}+S+S^{\prime}$ & $\phi_{1}$ & $S O(7):\left(s_{3}-1\right) \phi_{1}+2 S$ & $(17,19)$ & $S U\left(4-s_{3}\right)$ \\
\hline$(37)$ & $S O(8): s_{2} \phi_{1}+2 S+S^{\prime}$ & $\phi_{1}$ & $S O(7):\left(s_{2}-1\right) \phi_{1}+3 S$ & $(17,20)$ & $S U\left(3-s_{2}\right)$ \\
\hline$(38)$ & $S O(10):\left(2+s_{3}\right) \phi_{1}+S$ & $\phi_{1}$ & $S O(9):\left(1+s_{3}\right) \phi_{1}+S$ & $(21)$ & $S U\left(4-s_{3}\right)$ \\
\hline$(39)$ & $S O(10): 2 \phi_{1}+S$ & $\phi_{1}$ & $S O(9): \phi_{1}+S$ & $(22)$ & $G_{2}$ \\
\hline$(40)$ & $S O(10): \phi_{1}+S$ & $\phi_{1}$ & $S O(9): S$ & $(23)$ & $S O(7)$ \\
\hline NR & $S O(10): S$ & TDF & & & $S O(10)$ \\
\hline$(42)$ & $s_{3} \phi_{1}+s_{2} S+\left(2-s_{2}\right) S^{\prime}$ & $\phi_{1}$ & $S O(9):\left(s_{3}-1\right) \phi_{1}+2 S$ & $(24)$ & $S U\left(4-s_{3}\right)$ \\
\hline$(43)$ & $S O(10): 2 S$ & $2 S$ & & & $G_{2}$ \\
\hline$(44)$ & $S O(10): S+S^{\prime}$ & $S+S^{\prime}$ & $S U(5): \phi_{1}+\phi_{1}^{*}$ & (1) & $S U(4)$ \\
\hline$(45)$ & $\phi_{1}+\left(1+s_{2}\right) S+\left(2-s_{2}\right) S^{\prime}$ & $\phi_{1}$ & $S O(9): 3 S$ & $(25)$ & [ ] \\
\hline$(46)$ & $S O(10): 3 S$ & $2 S$ & $G_{2}: 2 \phi_{1}$ & $(60)$ & $S U(2)$ \\
\hline$(47)$ & $S O(10): 2 S+S^{\prime}$ & $2 S$ & $G_{2}: 2 \phi_{1}$ & $(16)$ & $S U(2)$ \\
\hline$(48)$ & $S O(12): s_{5} \phi_{1}+S$ & $\phi_{1}$ & $S O(11):\left(s_{5}-1\right) \phi_{1}+S$ & $26-28$ & $S U\left(6-s_{5}\right)$ \\
\hline $\mathbf{A}$ & $S O(12): 2 S$ & $S$ & $S U(6): \phi_{2}+\phi_{2}^{*}$ & (7) & $(S U(2))^{3}$ \\
\hline$(50)$ & $S O(12): S$ & $S$ & & & $S U(6)$ \\
\hline$(51)$ & $\phi_{1}+s_{2} S+\left(2-s_{2}\right) S^{\prime}$ & $\phi_{1}$ & $S O(11): 2 S$ & $(29)$ & {$[$ ] } \\
\hline$(52)$ & $S O(12): S+S^{\prime}$ & $S$ & $S U(6): \phi_{3}+\phi_{1}+\phi_{1}^{*}$ & (11) & $(S U(2))^{2}$ \\
\hline$(53)$ & $S O(14): s_{3} \phi_{1}+S$ & $\phi_{1}$ & $S O(13):\left(s_{3}-1\right) \phi_{1}+S$ & $(30,31)$ & $\left(S U\left(4-s_{3}\right)\right)^{2}$ \\
\hline \multirow[t]{2}{*}{ A } & $S O(14): S$ & $S$ & & & $G_{2} \times G_{2}$ \\
\hline & $\operatorname{SP}(2 \mathbf{n})$ theories & $<>$ & Higgs flow & entry & SYM \\
\hline$(55)$ & $S p(2 n): 2 k \phi_{1}: k \leq n$ & $2 \phi_{1}$ & $S p(2(n-1)): 2(k-1) \phi_{1}$ & $(1,55)$ & $S p(2(n-k))$ \\
\hline$(56)$ & $S p(2 n): \phi_{2}+2 \phi_{1}$ & $2 \phi_{1}$ & $S p(2(n-1)): \phi_{2}+2 \phi_{1}$ & $(1,56)$ & {[]} \\
\hline $\mathbf{A}$ & $S p(2 n): \phi_{2}$ & $\phi_{2}$ & & & $(S U(2))^{n}$ \\
\hline$(58)$ & $S p(4): 2 \phi_{2}$ & $\phi_{2}$ & $S O(4): \phi_{1}$ & $(32)$ & $S U(2)$ \\
\hline (59) & $S p(6): \phi_{3}+\phi_{1}$ & $\phi_{3}$ & $S U(3): \phi_{1}+\phi_{1}^{*}$ & (1) & $S U(2)$ \\
\hline
\end{tabular}


Table 3: All supersymmetric gauge theories with $\mu<\mu_{\text {adj }}$

\begin{tabular}{|l|l|c|l|c|l|}
\hline \hline & Exceptional groups & $<>$ & Higgs flow & entry & SYM \\
\hline$(60)$ & $G_{2}: s_{3} \phi_{1}$ & $\phi_{1}$ & $S U(3):\left(s_{3}-1\right)\left(\phi_{1}+\phi_{1}^{*}\right)$ & $(1)$ & $S U\left(4-s_{3}\right)$ \\
$(61)$ & $F_{4}: 2 \phi_{1}$ & $\phi_{1}$ & $S O(9): 2 \phi_{1}+S$ & $(21)$ & $S U(3)$ \\
$(62)$ & $F_{4}: \phi_{1}$ & $\phi_{1}$ & $S O(9): \phi_{1}$ & $(14)$ & $S O(8)$ \\
$(63)$ & $E_{6}: s_{2} \phi_{1}+\left(2-s_{2}\right) \phi_{1}^{*}$ & $\phi_{1}$ & $F_{4}: \phi_{1}$ & $(62)$ & $S O(8)$ \\
$(64)$ & $\left(1+s_{2}\right) \phi_{1}+\left(2-s_{2}\right) \phi_{1}^{*}$ & $\phi_{1}$ & $F_{4}: 2 \phi_{1}$ & $(61)$ & $S U(3)$ \\
$(65)$ & $E_{6}: \phi_{1}$ & $\phi_{1}$ & & & $F_{4}$ \\
$(66)$ & $E_{7}: 2 \phi_{1}$ & $\phi_{1}$ & $E_{6}: \phi_{1}+\phi_{1}^{*}$ & $(64)$ & $S O(8)$ \\
$(67)$ & $E_{7}: \phi_{1}$ & $\phi_{1}$ & & & $E_{6}$ \\
\hline
\end{tabular}

That's all folks

\section{Appendix B Dimension of the classical moduli space}

This appendix is devoted to some more mathematical results. We define Zariski topology, which was mentioned in Section. 2.2. Next, we make precise the notion of the dimension of the classical moduli space. We explain a subtlety encountered for theories without classical flat directions: $S O(10)$ with a spinor field and $S U(5)$ with $\mathbf{1 0}+\overline{\mathbf{5}}$. The same subtlety arises also for theories which have points in the moduli space with unbroken $S U(5)$ with $\mathbf{1 0}+\overline{\mathbf{5}}$, namely $S U(2 N+1)$ with $\boxminus$ and $(2 N-3) \bar{\square}$.

Let $V$ be an $n$ dimensional complex vector space, $V \cong \mathbf{C}^{n}$. In the Zariski topology, a closed subset $C \subseteq V$ is one that can be described as the set of zeros of a finite number of polynomials, i.e., $C=\left\{x \in \mathbf{C}^{n} \mid p_{\alpha}(x)=0, \alpha=1, \ldots s\right\} \equiv<p_{1}, \ldots, p_{s}>$. Open sets are those whose complements in $V$ are closed. The definition of Zariski closed sets satisfies the axioms of a topological space: $(i) V$ and $\emptyset$ are both open and closed, (ii) a finite union of closed sets is closed, and (iii) an arbitrary intersection of closed sets is closed. Property (iii) follows from Hilbert's basis theorem, according to which the zero set of an arbitrary set of polynomials agrees with the zero set of some finite set of polynomials.

Any non-empty Zariski open subset $O \subseteq V$ is dense in $V$, i.e., the smallest closed set containing $O$ is $V$ itself. For this reason, a property satisfied by every point in a Zariski open subset of a vector space $V$ is said to hold at "points at generic position". This makes precise the notion of "photons in the bulk of the moduli space" introduced in Section 2.2.

Another important property of non-empty Zariski open sets is that any two of them intersect non-trivially. The non-empty intersection is an open set, and therefore dense in $V$. An example of a Zariski closed set is the classical moduli space $\mathcal{M}_{c} \subseteq V, V$ the span of a 
basic set of gauge invariant polynomials $p(\phi)$ on the elementary fields $\phi . \mathcal{M}_{c}$ consists of the zeros of the polynomial constraints satisfied by the generators. The precise definition of the classical moduli space follows.

Having defined the Zariski topology we now discuss the classical moduli space and its dimension. Consider the vector space $U$ of microscopic fields $\phi^{i}$ in a gauge theory. Polynomials $p(\phi)$ on the chiral matter fields $\phi^{i}$ form an algebra. There is a representation of the gauge group $G_{r}$ on this algebra, namely

$$
g \cdot p(\phi)=p\left(g^{-1} \phi\right)
$$

which naturally extends to a representation of the complexification $G$ of $G_{r}$. If $G_{r}$ is the product of a compact, connected semi-simple Lie group with (possible) $U(1)$ factors, then the subalgebra of gauge invariant polynomials is finitely generated [25]. That means that a minimal set of basic gauge invariant polynomials $p_{1}(\phi), \cdots, p_{n}(\phi)$ can be found, in terms of which any gauge invariant polynomial $p(\phi)$ can be written as

$$
p(\phi)=\hat{p}\left(p_{1}(\phi), \ldots, p_{n}(\phi)\right)
$$

where $\hat{p}\left(p_{1}, \ldots, p_{n}\right)$ is a polynomial function. There is no unique choice for the basic invariants, but they can be chosen to be homogeneous, of degrees $d_{1} \leq d_{2} \leq \cdots \leq d_{n}$, and the sequence of degrees is uniquely determined by the $G$ representation [25]. In general, the basic invariants are constrained by a set of equations of the form

$$
k_{\alpha}\left(p_{1}, \ldots, p_{n}\right)=0
$$

meaning that when evaluating $p_{i}=p_{i}(\phi)$ in the above equation we get zero. The set of basic invariants together with the relations in Eq. B.3 is called classical moduli space, because their points are in one to one correspondence with D-flat configurations [4 6]. In some cases there are no constraints among the $p_{i}^{\prime} \mathrm{s}$, the algebra of gauge invariant polynomials is freely generated by $p_{1}, \ldots, p_{n}$.

We now consider the vector space $U$ of microscopic fields $\phi^{i}$. The set $C_{d} \subseteq U \cong \mathbf{C}^{n}$ of points whose orbits under the action of the group $G$ have dimension less than $d$ is closed. $C_{d}$ is closed because it is the set of zeros of all polynomials in $\phi$ obtained by taking the determinants of $d \times d$ submatrices of the $d_{G} \times n$ matrix expressing the action of $G$ on $\phi$. The $d_{G}$ columns of this matrix are $T_{A} \phi$, where $T_{A}$ are the generators of Lie $G, A=1, \ldots, d_{G}$. Taking $d$ to be equal to the maximum dimension of an orbit we learn that all points with orbits of maximal dimension form an open set, $\mathcal{O}_{1}^{\prime}$, the complement of $C_{\max }$. Therefore a generic configuration of VEVs of $\phi$ 's has orbits of maximal dimension, or the maximal number of broken generators.

It is shown in Ref. [11] that $\mathcal{O}_{1}^{\prime}$ contains an open subset with the property that at every point $\phi \in \mathcal{O}_{1}$, the subspace of unbroken generators is conjugate to a fixed subalgebra Lie $G_{*}$ 
of Lie $G$. The group $G_{*}$ is called the stabilizer at general positions. Note that we have not yet commented on the possibility of breaking $G$ to $G_{*}$ by a D-flat VEV. Whenever $G_{*}$ is reductivef, there exists a Zariski open, $G$ invariant set $\mathcal{O}_{2} \subset U$ containing closed orbits of $G$. This is equivalent to saying that every point in the open set $\mathcal{O}_{2}$ is gauge related to a D-flat point [4, 6]. As $\mathcal{O}_{1} \cap \mathcal{O}_{2} \neq \emptyset$ and open we conclude that whenever $G_{*}$ is reductive there is always a D-flat point that breaks $G$ to $G_{*}$. Moreover, since $\mathcal{O}_{1} \cap \mathcal{O}_{2}$ is open, the set of D-flat points breaking $G$ to $G_{*}$ is dense. We will label the minimal unbroken subgroup at the D-flat points $G_{P}$. When $G_{*}$ is reductive, $G_{P}=G_{*}$.

There are only two physical (gauge anomaly free) theories for which $G_{*}$ fails to be reductive: $S U(2 k+1)$ with an antisymmetric tensor and $2 k-3$ antifundamentals, and $S O(10)$ with a spinor, see Table $\Theta$. These are the only theories for which the $D$-flatness condition restricts the breaking of the gauge group to a subgroup $G_{P}$ of higher dimension than $G_{*}$. For all other theories $G_{*}=G_{P}$ and $\mathcal{O}_{1} \cap \mathcal{O}_{2}$ is a dense open set of points with a closed $G$ orbit of maximum dimension, from which follows that (Theorem 2 in Ref. [6])

$$
\operatorname{dim} \mathcal{M}_{c}=\operatorname{dim} U-\operatorname{dim} G+\operatorname{dim} G_{P}
$$

Note that the r.h.s. of (B.4) equals $\operatorname{dim} U$ minus the maximal dimension of a $G$ orbit in $U$. Note also that for the theories in Table 1 eq. (B.4) holds if we replace $G_{P}$ with $G_{*}$.

The dimension of $\mathcal{M}_{c}$ is much easier to compute using Eq. B.4 than its algebraic definition [25], which is a minimal number $r$ of gauge invariant polynomials $\chi^{1}(\phi), \ldots, \chi^{r}(\phi)$ for which every polynomial in a basic set of invariants $p^{1}(\phi), \ldots, p^{k}(\phi)$ satisfies an equation of the type

$$
\left(p^{j}\right)^{t}+\left(p^{j}\right)^{t-1} q_{1}\left(\chi^{1}, \ldots, \chi^{r}\right)+\cdots+\left(p^{j}\right) q_{t-1}\left(\chi^{1}, \ldots, \chi^{r}\right)+q_{t}\left(\chi^{1}, \ldots, \chi^{r}\right)=0 .
$$

The above equation makes precise the notion of "number of basic invariants minus number of independent constraints."

As an example, consider supersymmetric QCD with the number of colors equal to the number of flavors and the usual choice of basic invariants $M_{j}^{i}=Q^{i \alpha} \tilde{Q}_{\alpha j}, B=\operatorname{det} Q, \tilde{B}=$ $\operatorname{det} \tilde{Q}$, where $Q^{i \alpha}$ are the quarks and $\tilde{Q}_{j \beta}$ the anti-quarks. Here, $i, j$ are flavor indices, $i, j=1, \ldots, N$, and $\alpha, \beta=1, \ldots, N$ are the color ones. A set of $\chi^{\prime}$ 's is $\left\{M_{j}^{i}, \tilde{B}-B\right\}$. In fact

$$
\tilde{B}^{2}-(\tilde{B}-B) \tilde{B}-\operatorname{det} M=0 \quad B^{2}+(\tilde{B}-B) B-\operatorname{det} M=0,
$$

and it is easy to see that there is no smaller set of invariants satisfying $(\mathbb{B . 5})$ for supersymmetric QCD. This tells us that $\operatorname{dim} \mathcal{M}_{c}=N^{2}+1$, which agrees with (B.4), as $G_{P}$ is trivial. The definition and computation of dimensions when there is a tree level superpotential involves a number of additional subtleties [6].

\footnotetext{
${ }^{4}$ This means that every representation of $G_{*}$ can be broken up into irreducible blocks. This is always true if Lie $G_{*}$ is the sum of a semi-simple Lie algebra and (possible) $U(1)^{\prime} s$.
} 
Table 4: The only supersymmetric gauge theories with simple gauge group and non reductive stabilizer at general position $G_{*}$. $G_{P}$ is the unbroken subgroup at D-flat points where $G$ is maximally broken, and equals $G_{*}$ when $G_{*}$ is reductive. $d_{\mathcal{M}_{c}}$ is the dimension of the moduli space. In the last column we give a basic set of gauge invariants, its number equals $d_{\mathcal{M}_{c}}$ because there are no constraints among them. $\mathfrak{u}(n)$ denotes the Lie algebra of a unipotent group of dimension $n$, which is the "non-reductive piece" of $G_{*}$.

\begin{tabular}{|c|c|c|c|c|c|}
\hline$G$ & $\rho$ & $\operatorname{Lie}\left(G_{*}\right)$ & $\operatorname{Lie}\left(G_{P}\right)$ & $d_{\mathcal{M}_{c}}$ & Invariants \\
\hline \hline$S O(10)$ & $\operatorname{spin}$ & $\mathfrak{s o}(7)+\mathfrak{u}(8)$ & $\mathfrak{s o}(10)$ & 0 & no invariants \\
$S U(2 k+1)$ & $\boxminus+(2 k-3) \bar{\square}$ & $\mathfrak{s u}(2)+\mathfrak{u}(6)$ & $\mathfrak{s u}(5)$ & $(2 k-3)(k-2)$ & $A^{\alpha \beta} \bar{Q}_{\alpha}^{i} \bar{Q}_{\beta}^{j}$ \\
\hline
\end{tabular}

\section{References}

[1] I. Affleck, M. Dine, and N. Seiberg, Phys. Rev. Lett. 51, 1026 (1983); Nucl. Phys. B241, 493 (1984).

[2] D. Amati, K. Konishi, Y. Meurice, G. Rossi, and G. Veneziano, Phys. Rep. 162, 169 (1988).

[3] N. Seiberg, Phys. Rev. D49, 6857 (1994); Nucl. Phys. B435, 129 (1995).

[4] C. Procesi and G. Schwarz, Phys. Lett. 161B, 117 (1985).

[5] F. Buccella, J. Derendinger, S. Ferrara, and C. Savoy, Phys. Lett. 115B, 375 (1982); R. Gatto and G. Sartori, Phys. Lett. 157B, 389 (1985).

[6] G. Dotti and A. Manohar, Nucl. Phys. B518, 575 (1998).

[7] G. Dotti and A. Manohar, Phys. Rev. Lett. 80, 2758 (1998).

[8] C. Csáki and W. Skiba, Phys. Rev. D58, 045008 (1998).

[9] G.W. Schwarz, Inv. Math. 49, 167 (1978).

[10] O.M. Adamovich and E.O. Golovina, Sel. Math. Sov. 3, 183 (1983).

[11] A.G. Élashvili, Funct. Anal. Appl. 6, 44 (1972).

[12] E. Andreev, E. Vinberg, and A. Élashvili, Funct. Anal. Appl. 1, 257 (1968).

[13] C. Csáki, M. Schmaltz, and W. Skiba, Phys. Rev. Lett. 78, 799 (1997); Phys. Rev. D55, 7840 (1997). 
[14] B. Grinstein and D. Nolte, Phys. Rev. D57, 6471 (1998); Phys. Rev. D58, 045012 (1998).

[15] P. Cho, Phys. Rev. D57, 5214 (1998).

[16] K. Intriligator and N. Seiberg, Nucl. Phys. B444, 125 (1995).

[17] K. Intriligator and P. Pouliot, Phys. Lett. 353B, 471 (1995).

[18] I. Pesando, Mod. Phys. Lett. A10, 1871 (1995); S. Giddings and J. Pierre, Phys. Rev. D52, 6065 (1995); P. Pouliot, Phys. Lett. 353B, 471 (1995).

[19] V. Gatti and E. Viniberghi (sic), Adv. Math. 30, 137 (1978).

[20] C. Csáki and H. Murayama, hep-th/9804061.

[21] K. Intriligator, R. Leigh, and N. Seiberg, Phys. Rev. D50, 1092 (1994).

[22] J. Brodie, P. Cho, and K. Intriligator, Phys. Lett. 429B, 319 (1998).

[23] D. Kutasov, A. Schwimmer, and N. Seiberg, Nucl. Phys. B459, 455 (1996).

[24] K. Intriligator, R. Leigh, and M. Strassler, Nucl. Phys. B456, 567 (1995).

[25] R. Goodman and N. Wallach, Representation and Invariants of the Classical Groups, (Cambridge University Press, Cambridge, 1998). 